\title{
An Improved Corner Detection Algorithm Based on Harris Zeng Zhaoyang ${ }^{1, ~ a ~}$, Jiang Zhiqiang ${ }^{1, b}$, Chen Qiang ${ }^{1, \mathrm{c}}$, He Panfeng ${ }^{1, \mathrm{~d}}$ \\ ${ }^{1}$ The Academy of Equipment, Beijing, 101416, China \\ acyzeng@139.com , biang20062250105@163.com, \\ cbeckhamcq@126.com, dhepanfeng01@gmail.com
}

\begin{abstract}
Keywords: Corner detection; Harris corner detection; SUSAN corner detection; Eliminate false corners.
\end{abstract}

\begin{abstract}
In order to accurately extract corners from the image with high texture complexity, the paper analyzed the traditional corner detection algorithm based on gray value of image. Although Harris corner detection algorithm has higher accuracy, but there also exists the following problems: extracting false corners, the information of the corners is missing and computation time is a bit long. So an improved corner detection algorithm combined Harris with SUSAN corner detection algorithm is proposed, the new algorithm first use the Harris to detect corners of image, then use the SUSAN to eliminate the false corners. By comparing the test results show that the new algorithm to extract corners very effective, and better than the Harris algorithm in the performance of corner detection.
\end{abstract}

\section{Introduction}

The corner points in images contain large amounts of information, which can reflect the local features of image, and provide important information for further processing. The corner detection methods exist for achieving this are many, mainly they can be divided into two categories: one is based on the edges[1], the other is based on the grayscale changes[2].

The most widely used operators in the grayscale-based corner detection algorithm are SUSAN detector and Harris detector. The two methods are both having advantages and disadvantages, and applying to appropriate environments and conditions of its own. This paper is trying to make some improvements aiming at the weaknesses of Harris detector, making it better in extracting the corner points in images.

\section{Harris Corner Detector}

The basic idea of Harris corner detector[3] is to calculate grayscale changes of each pixel in the image. First, calculate the autocorrelation matrix M, then judge which one is feature point according to its eigenvalue. In this algorithm, if the eigenvalues are all greater than threshold, we take it as a feature point.

We define a corner point response function in Harris detector as follows:

$$
\mathrm{C}=\operatorname{DetM}-\mathrm{K} \times(\operatorname{trM})^{2} \text {. }
$$

Where DetM $=\lambda_{1} \times \lambda_{2}, \operatorname{trM}=\lambda_{1}+\lambda_{2}$, generally $\mathrm{K}$ lies in 0.04 and 0.06 .

The following are the specific steps involved in Harris corner detector[4]:

(1)Calculate the autocorrelation matrix $\mathrm{M}$ of each pixel:

$$
\mathrm{M}=\left[\begin{array}{ll}
\mathrm{A} & \mathrm{C} \\
\mathrm{C} & \mathrm{B}
\end{array}\right] \text {, where } \mathrm{A}=\left(\frac{\partial \mathrm{I}}{\partial \mathrm{x}}\right) \otimes \mathrm{w}, \mathrm{B}=\left(\frac{\partial \mathrm{I}}{\partial \mathrm{y}}\right) \otimes \mathrm{w}, \mathrm{C}=\left(\frac{\partial \mathrm{I}}{\partial \mathrm{x}} \frac{\partial \mathrm{I}}{\partial \mathrm{y}}\right) \otimes \mathrm{w}, \otimes \text { represents }
$$

convolution, w is Gauss window function.

By filtering the image with the level difference operator and vertical differential operator respectively, we can work out the gradient in $\mathrm{x}$ and $\mathrm{y}$ direction of the image I: 


$$
\frac{\partial \mathrm{I}}{\partial \mathrm{x}} \text { and } \frac{\partial \mathrm{I}}{\partial \mathrm{y}} \text {, that is } \frac{\partial \mathrm{I}}{\partial \mathrm{x}}=\mathrm{I} \otimes\left[\begin{array}{ccc}
-1 & 0 & 1 \\
-1 & 0 & 1 \\
-1 & 0 & 1
\end{array}\right], \frac{\partial \mathrm{I}}{\partial \mathrm{y}}=\mathrm{I} \otimes\left[\begin{array}{ccc}
-1 & -1 & -1 \\
0 & 0 & 0 \\
1 & 1 & 1
\end{array}\right]
$$

(2) Use Eq. 1 to calculate the corner response function $\mathrm{C}(\mathrm{x}, \mathrm{y})$ for each pixel point $(\mathrm{x}, \mathrm{y})$;

(3) Choose the pixel point with the maximum corner response function value as the feature point candidate;

(4) Set threshold value, and eliminate the point with $\mathrm{C}(\mathrm{x}, \mathrm{y})$ smaller than threshold.

\section{An Improved Harris Corner Detection}

Harris detector has a very accurate position precision with regard to corner points, and a relatively better result. However, Harris detector would lose part of corner point information when having a larger threshold value, and extract many false corner points when threshold value is smaller.

Use a circular mask to move in the image[5], if the pixel grayscale difference between the point in the mask and the nucleus of the mask is less than certain threshold, then we suppose that point and the nucleus have similar grayscale. And the areas composed of such point which satisfies the conditions above call USAN (Univalue Segment Assimilating Nucleus), as is shown in Fig. 1, when detecting the whole image, utilize a circular mask to traverse the whole image. When circular mask is completely on the background or target, we get the largest USAN area; when the mask approaches the edge of the target, the USAN area decreases gradually; when the mask nucleus is on the edge of the target, USAN area is very small; when the mask nucleus in the corner point, USAN area is smallest, This is SUSAN corner detector. According to this principle, we can not only detect corner points, but also judge whether the corner point is false. This paper will use this method to perform anti-false corner point processing to corner points extracted by Harris corner detector.

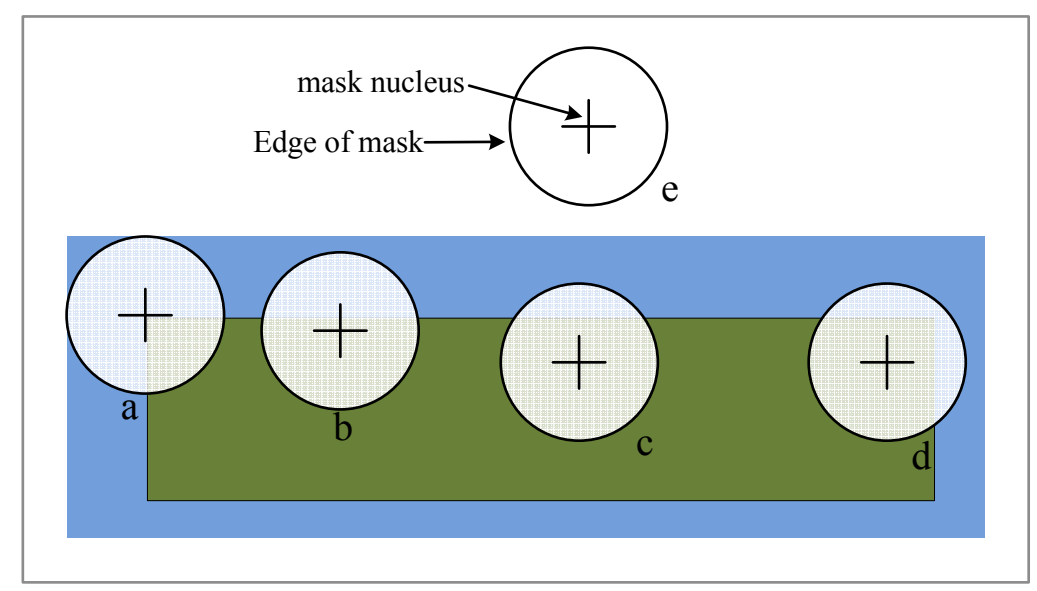

Fig. 1 USAN(Univalue Segment Assimilating Nucleus)

The following are detailed steps:

(1)First, use Harris detector to detect corner points in the image;

(2)Then construct a circular mask to transverse every corner point extracted from the image. Judge the similarity between the point in the region covered by the mask and the mask itself. The similarity comparison function is:

$$
\mathrm{C}(\mathrm{x}, \mathrm{y})=\exp \left\{-\left[\frac{\mathrm{I}(\mathrm{x}, \mathrm{y})-\mathrm{I}\left(\mathrm{x}_{0}, \mathrm{y}_{0}\right)}{\mathrm{t}}\right]^{6}\right\}
$$

Where, $\left(\mathrm{x}_{0}, \mathrm{y}_{0}\right)$ is the coordinate of the mask in the image, $(\mathrm{x}, \mathrm{y})$ is the coordinate of other points in the mask. $\mathrm{I}(\mathrm{x}, \mathrm{y})$ and $\mathrm{I}\left(\mathrm{x}_{0}, \mathrm{y}_{0}\right)$ are the grayscale of above two points, threshold $\mathrm{t}$ determines the maximum diversity of the similar two points, $\mathrm{C}$ denotes output result. 
(3)Use following formula to find the USAN value of the mask region:

$$
\mathrm{n}\left(\mathrm{x}_{0}, \mathrm{y}_{0}\right)=\sum_{(\mathrm{x}, \mathrm{y}) \neq\left(\mathrm{x}_{0}, \mathrm{y}_{0}\right)} \mathrm{C}(\mathrm{x}, \mathrm{y})
$$

(4)Compare $n$ with certain threshold, and calculate the response function regarding to image corner point.

$$
\mathrm{R}\left(\mathrm{x}_{0}, \mathrm{y}_{0}\right)=\left\{\begin{array}{cc}
\mathrm{g}-\mathrm{n}\left(\mathrm{x}_{0}, \mathrm{y}_{0}\right) & \mathrm{n}\left(\mathrm{x}_{0}, \mathrm{y}_{0}\right)<\mathrm{g} \\
0 & \text { otherw ise }
\end{array} .\right.
$$

Where, g represents threshold, half the area of the mask generally.

(5)Perform non-maximal suppression method. That is, choose one edge point as the center of the $3 * 3$ mask, and compare it with its eight other neighborhoods, keep the point with largest grayscale down.

In this way, we can eliminate the false corner point extracted by Harris detector, thus making the extracted point more reasonable, and its position more accurate.

\section{Simulations And Analysis}

The original image used in this experiment is the classic Lenna image in digital image processing. Separate using the Harris detection algorithm on Lenna image, the detected effect is shown in Fig. 2:

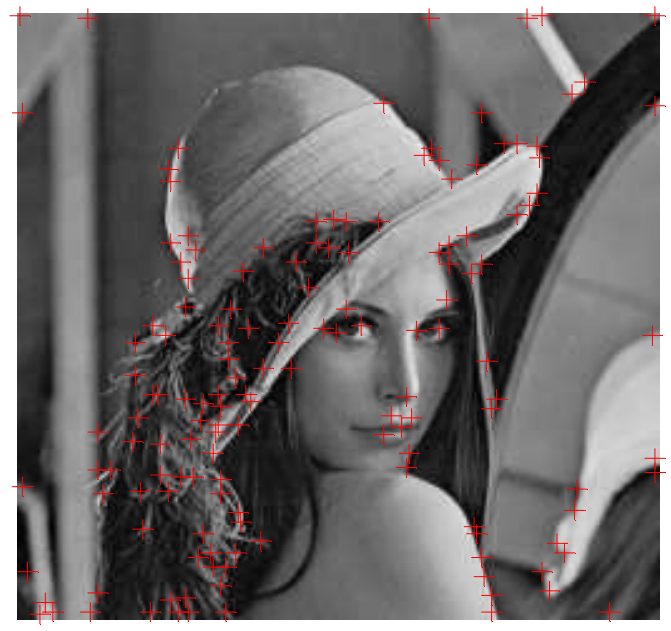

Fig. 2 The detected effect of Harris Operator

Separate using SUSAN Operator on the same image, the effect is shown in Fig. 3:

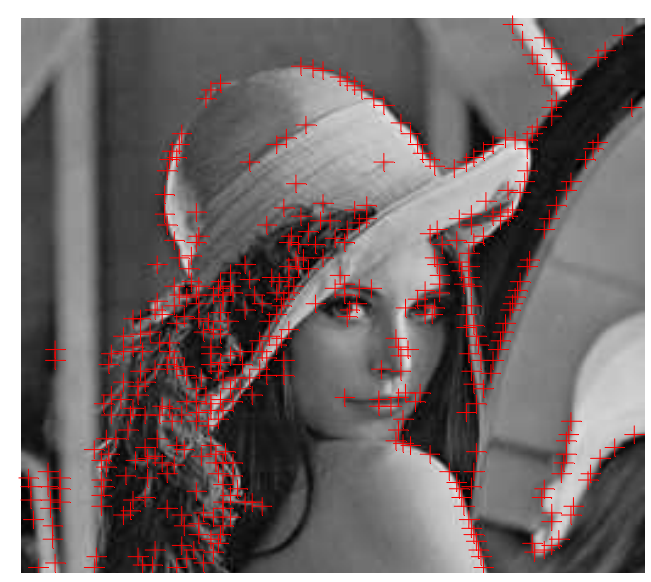

Fig. 3 The detected effect of SUSAN Operator 
Use the improved algorithm that's put forward in this paper to process this image, the effect is shown in Fig. 4:

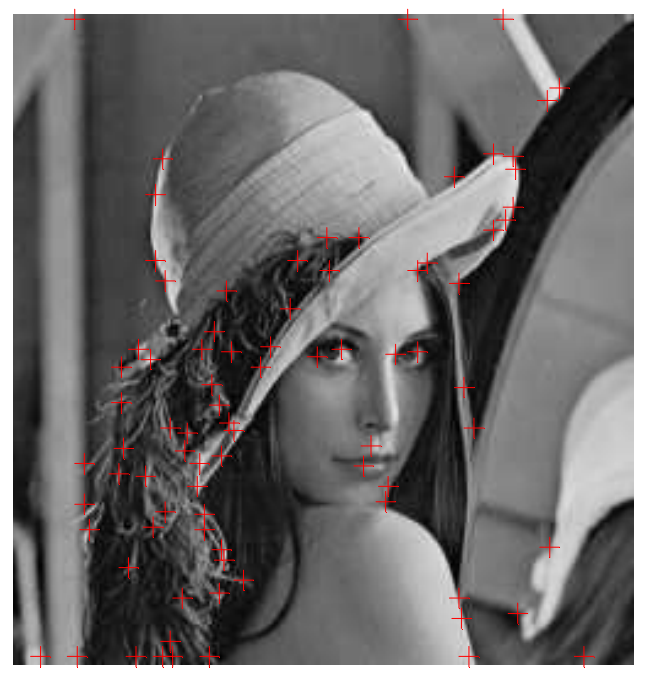

Fig. 4 The detected effect of improved algorithm

It can be seen in the simulation results that the corner points extracted by Harris corner detection algorithm are evenly distributed, corner points positioning accuracy, calculation is simple and easy to implement, however, in the extracted corner points existence pseudo-corner points. The corner points extracted by SUSAN corner detection algorithm are concentrated distributed, the effect is not very satisfactory, but, this method can effectively eliminated part of the pseudo-corner points. The improved algorithm in this paper combined with the Harris algorithm and the SUSAN algorithm achieved the ideal effect. Comparison of the number of corner points detected by the three corner detection algorithms and computation time of the three methods is shown in Table 1:

Table 1 Comparison of Algorithms

\begin{tabular}{llll}
\hline algorithms & Harris Operator & SUSAN Operator & Improved Algorithm \\
\hline $\begin{array}{l}\text { the number of corner } \\
\text { points }\end{array}$ & 149 & 439 & 83 \\
Computation time & $1.022006[\mathrm{~s}]$ & $1.005942[\mathrm{~s}]$ & $1.424114[\mathrm{~s}]$ \\
\hline
\end{tabular}

\section{Conclusions}

According to the above simulation results, the effect of SUSAN corner detection operator isn't satisfaction, the threshold need to reconfigure when the image changed, the degree of automation is low, but this algorithm's computation time is the least. Harris corner detection algorithm can better extract corner points of the image and the corner points positioning accuracy, but the operation time longer than SUSAN algorithm and pseudo-corner points will be extracted when the threshold sets smaller. The improved algorithm fully combined advantages of both algorithms, it's not only gets a more precise corner points pixel position but also effectively eliminates a majority of the pseudocorner points. The test results show that the new algorithm to extract corners very effective, and better than other algorithm in the performance of corner detection. 


\section{References}

[1] F Schaffalitzky and A Zisserman. Viewpoint invariant texture matching and wide baseline stereo[J].IEEE, (2001).

[2] C.Schmid,R.Mohr and C.Bauckhage. ComParing and evaluating interest Points.The 6th International Conference on ComPuter Vision.(1998)230-235.

[3] Guo Feifei.Feature-based image registration and its application.College of Mathematics and Statistics of Chongqing University. (2010).

[4] Chai Jiali.Fueature points extraction and fast matching of stereo vision images.Hebei University. (2010).

[5] Mao Yanming,Lan Meihui,Wang Yunqiong.An improved corner detection method based on harris.Computer technology and development 19 (2009)130-133.. 